\title{
Éclosion communautaire et nosocomiale de Legionella pneumophila à Montréal, Québec, 2019
}

\author{
Geneviève Cadieux ${ }^{1 *}$, Julie Brodeur ${ }^{1}$, Félix Lamothe ${ }^{1}$, Cindy Lalancette ${ }^{2}$, Pierre A. Pilon ${ }^{1}$, \\ David Kaiser ${ }^{1}$, Éric Litvak ${ }^{1}$
}

\section{Résumé}

Objectifs : Décrire l'enquête d'une éclosion communautaire de Legionella pneumophila de sérogroupe 1, avec des sous-agrégats dans une résidence privée pour aînés et un hôpital à Montréal, QC, et les principaux défis rencontrés.

Méthodes : Il y a eu 14 cas d'infection à L. pneumophila de sérogroupe 1 dont la date de l'apparition des symptômes se situait entre le 7 juin et le 21 août 2019. L'enquête environnementale comprenait l'échantillonnage des tours de refroidissement à l'eau (TRE) et d'autres sources potentielles. Un typage des isolats cliniques et environnementaux a été effectué. Les interventions de santé publique ont inclus des ordonnances de décontamination des tours de refroidissement à l'eau et la communication avec les cliniciens.

Résultats : Onze (79\%) des 14 cas étaient immunodéprimés ou immunodéficients. La plupart $(13,93 \%)$ ont été diagnostiqués à l'aide d'un test d'antigène urinaire, et cinq (36 \%) ont subi une culture. Deux sous-agrégats ont été détectés : trois cas dans une résidence privée pour aînés et quatre cas sur un étage d'un hôpital. Les résultats du typage suggèrent que la même L. pneumophila sérogroupe 1 pourrait avoir causé l'éclosion communautaire et les deux sousagrégats. Une source environnementale correspondante n'a pas été identifiée.

Conclusion : Alors que le typage des isolats cliniques suggérait une source environnementale commune, notre enquête n'a pas réussi à déterminer cette source. Les futures enquêtes sur les éclosions pourraient bénéficier d'un plus grand nombre d'isolats cliniques pour le typage, de registres locaux des sources d'aérosolisation de l'eau autres que les tours de refroidissement à l'eau et d'un accès en continu à tous les résultats de la surveillance de routine des tours de refroidissement à l'eau et aux isolats de L. pneumophila pour le typage.
Citation proposée : Cadieux G, Brodeur J, Lamothe F, Lalancette C, Pilon PA, Kaiser D, Litvak E. Éclosion mixte et nosocomial de Legionella pneumophila à Montréal, Québec, 2019. Relevé des maladies transmissibles au Canada 2020;46(7/8):246-54. https://doi.org/10.14745/ccdr.v46i78a01f

Mots-clés : Legionella, Legionella pneumophila, légionellose, maladie du légionnaire, éclosions de maladie, contrôle des maladies transmissibles, santé publique, microbiologie de l'eau, qualité de l'eau
Cette oeuvre est mise à la disposition selon les termes de la licence internationale Creative Commons Attribution 4.0

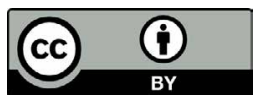

Affiliations

${ }^{1}$ Direction régionale de santé publique de Montréal, Montréal, QC

2 Laboratoire de santé publique du Québec, Sainte-Anne-deBellevue, QC

\section{${ }^{\star}$ Correspondance :}

genevieve.cadieux.ccsmt|@ssss. gouv.qc.ca

\section{Introduction}

L'infection à Legionella sp. est devenue une maladie à déclaration obligatoire dans la province de Québec $(Q C)$ en 1987. L'incidence de la légionellose est en augmentation depuis 2006 (1). En 2012, une éclosion de Legionella pneumophila de sérogroupe 1 au Québec liée à une tour de refroidissement à l'eau (TRE) a provoqué 183 cas et 13 décès $(1,2)$. À la suite de cette éclosion, la province a introduit une réglementation qui exige que toutes les tours de refroidissement à l'eau soient enregistrées auprès de la Régie du bâtiment du Québec (RBQ) (3) et fassent l'objet d'un entretien régulier et d'une surveillance mensuelle pour la L. pneumophila (1). L'échantillonnage des TRE doit être effectué conformément aux directives provinciales (4), et les résultats doivent être soumis mensuellement à la RBQ. Le règlement exige que les propriétaires de TRE appliquent des mesures d'atténuation lorsque les résultats se situent entre 10000 et 999999 unités formant des colonies (UFC)/L.

De plus, depuis juillet 2014, les résultats de la surveillance de L. pneumophila de 1000000 de UFC/L ou plus doivent être 
la DRSP sont enquêtés à l'aide d'un questionnaire provincial standard (3); des données sont recueillies sur les facteurs de risque (notamment le diabète, les maladies pulmonaires chroniques, les maladies cardiovasculaires, les maladies rénales chroniques, l'affaiblissement de l'immunité lié à la maladie ou aux médicaments, le cancer, la chimiothérapie ou la radiothérapie au cours des six derniers mois, le tabagisme, la consommation d'alcool), la présentation clinique, les résultats des tests de diagnostic, les complications; et les expositions potentielles pendant la période d'incubation (y compris les voyages, les soins de santé, les spas/piscines, les fontaines décoratives, les fontaines d'eau potable, les épiceries, les serres, les parcs aquatiques, les problèmes ou travaux sur le réseau de distribution d'eau potable, les problèmes de plomberie, les équipements produisant des aérosols, la température du chauffe-eau, les traitements dentaires, les expositions professionnelles et sur le lieu de travail). Pour les cas associés à un agrégat spatio-temporels ou à une éclosion, des renseignements détaillés sont recueillis sur tous les sites visités pendant la période d'incubation (3). Les enquêteurs de la santé publique demandent régulièrement aux médecins traitants d'envisager de prescrire aussi une culture d'expectorations pour la Legionella pour les patients dont le test d'antigène urinaire est positif.

Un cas confirmé lié à l'éclosion était défini comme un cas répondant à la définition provinciale de la légionellose (3), ayant une culture positive pour la L. pneumophila de sérogroupe 1 avec dont le typage génétique correspondait aux autres cas faisant partie de l'éclosion, et ayant résidé ou travaillé dans un rayon de $3 \mathrm{~km}$ de l'épicentre pendant sa période d'incubation. Un cas probable lié à l'éclosion correspondait à la définition provinciale de cas de légionellose (3); il n'avait qu'un test $d$ 'antigène urinaire positif pour la L. pneumophila de sérogroupe 1; et il avait résidé ou travaillé dans un rayon de $3 \mathrm{~km}$ de l'épicentre pendant sa période d'incubation. La période d'incubation habituelle de 2 à 10 jours a été prolongée jusqu'à 21 jours pour les patients immunodéprimés et immunodéficients; la période d'incubation pour les quatre premiers cas qui ont déclenché l'enquête sur les groupes s'étendait du 24 mai au 28 juin 2019.

Lors de l'enquête des cas, deux sous-agrégats ont été détectés : trois cas dans une résidence privée pour aînés et quatre cas nosocomiaux sur le même étage d'un hôpital. Les résidents de la résidence privée pour aînés vivaient sur différents étages et dans différentes tours. Les cas nosocomiaux avaient été hospitalisés au même étage pendant toute la période d'incubation (plus de 21 jours).

\section{Enquête environnementale}

La tour de refroidissement à l'eau avec des niveaux de L. pneumophila de sérogroupe 1 de 1000000 UFC/L ou plus la plus proche de l'épicentre pendant la période d'incubation se trouvait à environ $8 \mathrm{~km}$, il était donc plus probable qu'une TRE plus proche avec une concentration plus faible de 
L. pneumophila puisse être la source. Le centre géographique approximatif des quatre cas a été identifié, et un rayon de $4 \mathrm{~km}$ a été tracé autour de ce centre (par mesure de précaution, $1 \mathrm{~km}$ a été ajouté au rayon de $3 \mathrm{~km}$ recommandé par des lignes directrices provinciales) (3). La vitesse et la direction du vent pendant la période d'incubation ont été analysées. La DRSP a demandé à la $\mathrm{RBQ}$ les résultats des tests mensuels de L. pneumophila de mai à juillet pour toutes les 59 TRE (38 sites) dans cette zone (3). L'examen manuel de ces résultats a permis d'identifier deux TRE dont la concentration de L. pneumophila de sérogroupe 1 était inférieure à 1000000 UFC/L, 16 avec des résultats manquants et aucun montrant une "flore interférente » (qui pourrait cacher la L. pneumophila).

Après qu'un cinquième cas de L. pneumophila de sérogroupe 1 dans la même zone géographique ait été déclaré le 12 juillet, la DRSP a émis une ordonnance de santé publique à la RBQ afin qu'elle obtienne des échantillons d'eau pour retester les 18 TRE présentant des résultats anormaux ou manquants. La RBO suit la directive provinciale pour l'échantillonnage des TRE (4); la procédure d'échantillonnage est la même pour la surveillance mensuelle de routine que pour les éclosions : un seul échantillon d'eau de $1 \mathrm{~L}$ est prélevé sur un site représentatif après avoir laissé l'eau couler pendant au moins 30 secondes pour purger l'eau stagnante.

À la suite de la déclaration de nouveaux cas en lien avec l'agrégat, l'épicentre et la zone de $4 \mathrm{~km}$ de rayon autour de celui-ci ont été légèrement ajustés. La DRSP a demandé à la RBQ de mettre à jour les résultats de la surveillance de la L. pneumophila pour toutes les TRE situées dans la zone les 19 et 31 juillet et le 12 août. Le 26 août, comme aucune source n'avait encore été identifiée, la DRSP a demandé que tous les isolats disponibles provenant de TRE situées dans un rayon de $12 \mathrm{~km}$ de l'épicentre et dont les résultats de la surveillance mensuelle de Legionella étaient de 1000000 UFC/L ou plus durant la période d'incubation des cas soient envoyés au laboratoire pour typage génétique.

En parallèle, sur la base des directives provinciales (3) et de la littérature scientifique (7-11), la DRSP a accédé aux bases de données disponibles concernant les travaux sur le réseau de distribution d'eau potable et les chantiers de construction entravant la circulation routière ainsi qu'aux images satellites pour rechercher d'autres sources potentielles d'aérosolisation de L. pneumophila de sérogroupe 1 dans un rayon de $2 \mathrm{~km}$ autour de l'épicentre. Au cours d'une visite sur le terrain le 7 août, des échantillons d'eau et/ou de biofilm ont été recueillis auprès d'un dispositif de décapage de peinture à haute pression et d'un tuyau d'arrosage utilisé pour compacter le sol sur un site d'excavation. Des échantillons d'eau provenant du système d'irrigation d'un terrain de golf (i.e. des arroseurs dans trois zones distinctes les plus proches des cas) ont été obtenus le 15 août.
Des enquêtes environnementales distinctes ont également été menées pour les deux sous-agrégats de cas de la résidence privée pour aînés et de l'hôpital. La DRSP a examiné les registres d'entretien de la piscine chauffée et du spa ainsi que les températures du réservoir d'eau chaude de la résidence privée pour aînés. Des échantillons d'eau du réservoir d'eau chaude, de la piscine chauffée, du spa et de la fontaine décorative extérieure ainsi que de l'eau potable des appartements distincts de deux des résidents qui avaient été infectés ont été prélevés le 25 juillet.

L'équipe de prévention et de contrôle des infections de l'hôpital a enquêté sur les sources potentielles à l'étage touché. Des échantillons d'eau/biofilm ont été prélevés sur les éviers, les robinets et les pommes de douche les 24 juillet et 13 août, et sur une machine à glace, un réfrigérateur, une machine à nettoyer les planchers et un tuyau d'arrosage extérieur le 13 août.

\section{Analyses de laboratoire d'échantillons cliniques et environnementaux}

Les hôpitaux établissant le diagnostic ont effectué une première analyse des échantillons cliniques; ces échantillons ou des isolats ont ensuite été transmis au laboratoire provincial de santé publique pour confirmation et typage. Sur les échantillons cliniques, les méthodes de confirmation de routine comprenaient un test de réaction en chaîne à la polymérase (PCR) en temps réel développé localement et ciblant les gènes ssrA, mip et wzm; sur les isolats cliniques, la confirmation a été effectuée en utilisant la désorption-ionisation par impact laser assistée par matrice par temps de vol (MALDI-ToF, Bruker, IVD 3,2) et le sérogroupe 1 a été identifié par un test d'agglutination sur lame avec des antisérums de la L. pneumophila de sérogroupe 1 (Denka Seiket, Japon). Le génotypage a été effectué selon le protocole du Groupe de travail européen sur les infections à Legionella (EWGLI) $(12,13)$.

Le Centre d'expertise en analyses environnementales du Québec (CEAEQ) a effectué les cultures et les analyses quantitatives en temps réel par PCR (L. pneumophila de sérogroupe 1) des échantillons d'eau des TRE obtenus en réponse à l'ordonnance de santé publique. Les analyses d'échantillons environnementaux autres que les TRE ont été effectuées par divers laboratoires commerciaux accrédités par le groupe CSA (L. pneumophila qPCR) et le CEAEQ (culture de Legionella). Tous les isolats environnementaux de la $L$. pneumophila de sérogroupe 1 ont été envoyés au laboratoire provincial de santé publique pour être typés.

\section{Interventions}

Par mesure de précaution, une ordonnance de décontamination, prenant effet immédiatement, a été émise à tous les propriétaires/gérants de TRE ayant obtenu un résultat positif pour la L. pneumophila (tout résultat quantifiable). L'équipe de santé environnementale de la DRSP a assuré un suivi auprès de chaque propriétaire/gestionnaire de TRE pour s'assurer que 
la décontamination était réalisée le plus rapidement possible. L'efficacité de la décontamination a été vérifiée à l'aide de nouveaux prélèvements des TRE entre 2 à 7 jours après la décontamination, conformément au protocole provincial (4). Un appel à la vigilance a été envoyé aux cliniciens de Montréal pour leur rappeler de considérer la légionellose dans leur diagnostic différentiel chez les patients à risque présentant une pneumonie et d'ordonner une culture d'expectorations pour la recherche de la Legionella afin de faciliter l'enquête de santé publique. L'éclosion a été déclarée terminée le 18 octobre, 45 jours après que le dernier cas ait été déclaré à la santé publique.

\section{Résultats}

\section{Enquête épidémiologique}

Un total de 14 cas de L. pneumophila de sérogroupe 1 ont fait partie de l'éclosion, la maladie s'étant déclarée entre le 7 juin et le 21 août 2019 (figure 1 et tableau 1). Parmi les 14 cas, dix étaient des hommes, l'âge médian était de 68 ans, 11 étaient immunodéprimés ou immunodéficients, quatre ont dû être admis dans une unité de soins intensifs, deux ont été intubés et un est décédé. Six cas vivaient dans des domiciles privés et trois dans la même résidence privée pour aînés (dans des tours différentes et à des étages différents); quatre étaient hospitalisés au même étage de l'hôpital pendant la durée de leur période $d$ 'incubation et un vivait dans une résidence pour personnes autonomes recevant des traitements contre le cancer, située près de l'hôpital.

\section{Figure 1 : Courbe épidémique de l'éclosion de} légionellose à Montréal, 2019

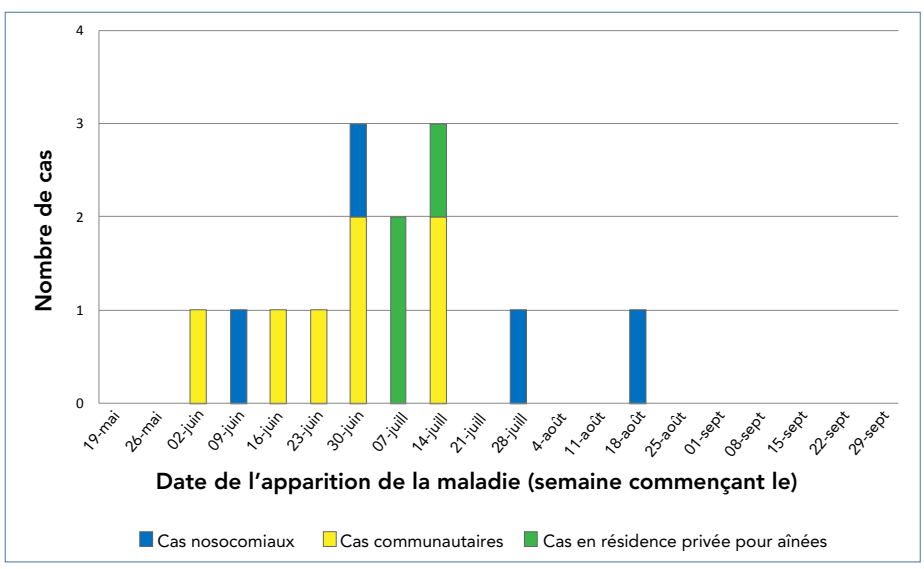

Treize cas ont été initialement diagnostiqués grâce à un test d'antigène urinaire positif; des cultures d'expectorations positives ont ensuite été obtenues pour quatre d'entre eux (tableau 1). Un patient anurique a été initialement diagnostiqué grâce à une culture d'expectorations.

À 63,8 ans (écart-type : 17,6; médiane : 68 ans), les cas liés à l'éclosion étaient généralement plus jeunes que les autres cas d'infection à L. pneumophila de sérogroupe 1 montréalais de
2019 dont l'âge moyen était de 69,1 ans (écart-type : 11,7; médiane : 65 ans). Une proportion plus importante présentait des comorbidités, notamment une immunité compromise ou une immunosuppression (voir tableau 2). Un examen détaillé des informations recueillies lors des enquêtes réalisées auprès cas n'a pas permis de mettre en évidence des expositions communes entre les cas autres que le lieu de résidence.

\section{Enquête environnementale}

Un total de 18 TRE dont les résultats de suivi mensuel étaient anormaux ou manquants ont été initialement échantillonnés sur ordonnance de la DRSP entre le 13 et le 18 juillet. Les échantillons ont d'abord été analysés pour la $L$. pneumophila par PCR quantitative et, en cas de résultat positif, par culture (tableau 3). Un résultat de culture quantifiable positif n'a été obtenu que pour une seule TRE (TRE-A). L'examen des résultats de la surveillance mensuelle mis à jour le 12 août a permis d'identifier trois TRE dont les résultats de culture étaient anormaux en juillet; les isolats de deux TRE étaient encore disponibles (TRE-B et TRE-C), mais ceux de la troisième avaient déjà été détruits. Une ordonnance de ré-échantillonnage a été émise, mais l'échantillon s'est révélé négatif. Douze autres TRE situées entre 4 et $12 \mathrm{~km}$ de l'épicentre ont obtenu des résultats de surveillance mensuelle pour la L. pneumophila de 1000000 UFC/L ou plus pendant la période d'incubation combinée de nos cas. Le laboratoire provincial de santé publique a procédé au typage des 15 isolats quantifiables de cultures de L. pneumophila de sérogroupe 1.

Les échantillons provenant de toutes les autres sources communautaires extérieures potentielles ont testés négatifs pour la L. pneumophila lors de l'analyse par PCR quantitative. Les échantillons de la résidence privée pour aînés (réservoir d'eau chaude, piscine chauffée, spa, fontaine décorative extérieure et eau potable) étaient tous négatifs pour la $L$. pneumophila lors de l'analyse par PCR quantitative. Tous les échantillons prélevés à l'hôpital le 24 juillet (15 échantillons) et les 13 et 14 août (19 échantillons) se sont révélés négatifs (25 échantillons) ou présentaient des taux de $L$. pneumophila inférieurs à la limite de quantification (9 échantillons) lors de l'analyse par PCR quantitative.

\section{Typage des isolats cliniques et environnementaux}

Au total, cinq isolats cliniques étaient disponibles pour le typage (tableau 4); tous les allèles séquencés correspondaient, ce qui suggère une source commune. Quatre cas présentaient six ou sept allèles séquencés, et tous les allèles séquencés correspondaient les uns aux autres; deux cas provenaient de I'hôpital, un de la résidence privée pour aînés, l'autre de la communauté. Seuls deux allèles du cas de la résidence pour personnes autonomes recevant des traitements contre le cancer ont pu être séquencés; les deux correspondaient aux autres cas. Le typage du le seul cas entièrement typé (tableau 4, ID du 
Tableau 1 : Cas de Legionella pneumophila de sérogroupe 1 liés à l'éclosion à Montréal, 2019

\begin{tabular}{|c|c|c|c|c|c|c|c|c|c|}
\hline $\begin{array}{l}\text { Identification } \\
\text { du cas }\end{array}$ & $\begin{array}{l}\text { Date de } \\
\text { déclaration }\end{array}$ & $\begin{array}{c}\text { Date de } \\
\text { l'apparition } \\
\text { de la } \\
\text { maladie }\end{array}$ & Sexe & $\begin{array}{c}\text { Groupe } \\
\text { d'âge } \\
\text { (années) }\end{array}$ & Facteurs de risque & $\begin{array}{c}\text { Test } \\
d^{\prime} \text { antigène } \\
\text { urinaire }\end{array}$ & $\begin{array}{c}\text { Culture } \\
\text { d'expectorations/ } \\
\text { LBA }\end{array}$ & $\begin{array}{l}\text { Évolution } \\
\text { clinique }\end{array}$ & Exposition \\
\hline 1 & 2019-06-13 & 2019-06-07 & $\mathrm{F}$ & $85-89$ & $\begin{array}{l}\text { Maladie pulmonaire } \\
\text { chronique, } \\
\text { immunosuppression, } \\
\text { cancer, tabagisme }\end{array}$ & Positif & NE & Hospitalisation & $\begin{array}{l}\text { Domicile } \\
\text { privé }\end{array}$ \\
\hline 2 & 2019-07-03 & $2019-06-26$ & $M$ & $25-29$ & $\begin{array}{l}\text { Immunosuppression, } \\
\text { cancer }\end{array}$ & Positif & NE & Hospitalisation & $\begin{array}{l}\text { Domicile } \\
\text { privé }\end{array}$ \\
\hline 3 & 2019-07-05 & 2019-06-30 & $M$ & $65-69$ & $\begin{array}{l}\text { Diabète, maladie rénale } \\
\text { chronique, tabagisme, } \\
\text { consommation } \\
\text { d'alcool supérieure aux } \\
\text { recommandations pour } \\
\text { une consommation à } \\
\text { moindre risque }\end{array}$ & Positif & Positif & $\begin{array}{l}\text { Hospitalisation, } \\
\text { séjour en } \\
\text { soins intensifs, } \\
\text { intubation }\end{array}$ & $\begin{array}{l}\text { Domicile } \\
\text { privé }\end{array}$ \\
\hline 4 & 2019-07-09 & 2019-06-18 & $M$ & $65-69$ & $\begin{array}{l}\text { Diabète, } \\
\text { immunosuppression, } \\
\text { maladie rénale } \\
\text { chronique, tabagisme }\end{array}$ & Positif & NE & $\begin{array}{l}\text { Hospitalisation, } \\
\text { séjour en } \\
\text { soins intensifs, } \\
\text { intubation }\end{array}$ & $\begin{array}{l}\text { Domicile } \\
\text { privé }\end{array}$ \\
\hline 5 & 2019-07-12 & 2019-07-10 & $\mathrm{F}$ & $75-79$ & $\begin{array}{l}\text { Diabète, maladie } \\
\text { cardiovasculaire, maladie } \\
\text { rénale chronique, } \\
\text { immunosuppression, } \\
\text { cancer, consommation } \\
\text { d'alcool supérieure aux } \\
\text { recommandations pour } \\
\text { une consommation à } \\
\text { moindre risque }\end{array}$ & Positif & $\mathrm{NE}$ & Hospitalisation & $\begin{array}{l}\text { Résidence } \\
\text { privée pour } \\
\text { aînés }\end{array}$ \\
\hline 6 & 2019-07-15 & 2019-07-02 & $\mathrm{F}$ & $30-34$ & $\begin{array}{l}\text { Immunosuppression, } \\
\text { tabagisme (cigarettes) }\end{array}$ & Positif & $\mathrm{NE}$ & Hospitalisation & $\begin{array}{l}\text { Domicile } \\
\text { privé }\end{array}$ \\
\hline 7 & 2019-07-19 & 2019-07-14 & $\mathrm{F}$ & $80-84$ & $\begin{array}{l}\text { Maladies } \\
\text { cardiovasculaires, } \\
\text { maladies rénales } \\
\text { chroniques }\end{array}$ & Positif & $\mathrm{NE}$ & Hospitalisation & $\begin{array}{l}\text { Domicile } \\
\text { privé }\end{array}$ \\
\hline 8 & 2019-07-19 & $2019-07-10$ & M & $75-79$ & $\begin{array}{l}\text { Diabète, maladie } \\
\text { cardiovasculaire, } \\
\text { tabagisme (cigarettes) }\end{array}$ & Positif & NE & Hospitalisation & $\begin{array}{l}\text { Résidence } \\
\text { privée pour } \\
\text { aînés }\end{array}$ \\
\hline $9^{a}$ & $2019-07-22$ & 2019-07-01 & M & $55-59$ & $\begin{array}{l}\text { Immunosuppression, } \\
\text { tabagisme (cigarettes) }\end{array}$ & Positif & Positif & Hospitalisation & Hôpital \\
\hline 10 & $2019-07-22$ & 2019-07-15 & $M$ & $60-64$ & $\begin{array}{l}\text { Diabète, maladie } \\
\text { cardiovasculaire, maladie } \\
\text { rénale chronique, } \\
\text { immunosuppression }\end{array}$ & $\begin{array}{l}\mathrm{NE} \\
\text { (anurique) }\end{array}$ & Positif & $\begin{array}{l}\text { Hospitalisation, } \\
\text { séjour en } \\
\text { soins intensifs, } \\
\text { intubations }\end{array}$ & Hôpital \\
\hline 11 & $2019-07-22$ & $2019-07-20$ & $M$ & $60-64$ & $\begin{array}{l}\text { Immunosuppression, } \\
\text { cancer }\end{array}$ & Positif & Positif & $\begin{array}{l}\text { Hospitalisation, } \\
\text { séjour en } \\
\text { soins intensifs, } \\
\text { intubation }\end{array}$ & $\begin{array}{l}\text { Résidence } \\
\text { pour } \\
\text { personnes } \\
\text { autonomes } \\
\text { recevant des } \\
\text { traitements } \\
\text { contre le } \\
\text { cancer }\end{array}$ \\
\hline 12 & $2019-07-24$ & 2019-07-16 & $M$ & $70-74$ & $\begin{array}{l}\text { Diabète, maladie } \\
\text { cardiovasculaire, } \\
\text { immunosuppression, } \\
\text { tabagisme (cigarettes) }\end{array}$ & Positif & Positif & Hospitalisation & $\begin{array}{l}\text { Résidence } \\
\text { privée pour } \\
\text { aînés }\end{array}$ \\
\hline 13 & 2019-08-12 & 2019-08-03 & $M$ & $75-79$ & $\begin{array}{l}\text { Diabète, maladie } \\
\text { pulmonaire chronique, } \\
\text { maladie cardiovasculaire, } \\
\text { immunosuppression, } \\
\text { tabagisme (cigarettes) }\end{array}$ & Positif & NE & Hospitalisation & Hôpital \\
\hline 14 & 2019-09-03 & 2019-08-21 & $M$ & $50-54$ & Immunosuppression & Positif & NE & Hospitalisation & Hôpital \\
\hline
\end{tabular}

Abréviations : $F$, féminin; LBA, lavage bronchoalvéolaire; $M$, masculin; NE, non effectué

a Ne réside pas à Montréal 
Tableau 2 : Caractéristiques des cas de Legionella pneumophila de sérogroupe 1 à Montréal, 2019

\begin{tabular}{|c|c|c|c|c|}
\hline \multirow[t]{2}{*}{ Caractéristiques } & \multicolumn{2}{|c|}{$\begin{array}{l}\text { Cas dans cette } \\
\text { éclosion } \\
(\mathrm{N}=14)\end{array}$} & \multicolumn{2}{|c|}{$\begin{array}{c}\text { Autres cas à } \\
\text { Montréal en } 2019 \\
(\mathrm{~N}=41)\end{array}$} \\
\hline & $n$ & $\%$ & $n$ & $\%$ \\
\hline \multicolumn{5}{|l|}{ Groupe d'âge (années) } \\
\hline $25-34$ & 2 & 14 & 0 & 0 \\
\hline $35-44$ & 0 & 0 & 0 & 0 \\
\hline $45-54$ & 1 & 7 & 3 & 7 \\
\hline $55-64$ & 3 & 21 & 16 & 39 \\
\hline $65-74$ & 3 & 21 & 10 & 24 \\
\hline $75-84$ & 4 & 29 & 5 & 12 \\
\hline 85 ans ou plus & 1 & 7 & 7 & 17 \\
\hline \multicolumn{5}{|c|}{ Comorbidités (autodéclarées) } \\
\hline Diabète & 7 & 50 & 12 & 29 \\
\hline $\begin{array}{l}\text { Maladie pulmonaire } \\
\text { chronique }\end{array}$ & 3 & 21 & 6 & 15 \\
\hline $\begin{array}{l}\text { Maladie } \\
\text { cardiovasculaire }\end{array}$ & 6 & 43 & 21 & 51 \\
\hline $\begin{array}{l}\text { Maladie rénale } \\
\text { chronique }\end{array}$ & 5 & 36 & 5 & 12 \\
\hline Immunosuppression & 11 & 79 & 6 & 15 \\
\hline \multicolumn{5}{|c|}{ Autres facteurs de risque (autodéclarés) } \\
\hline Tabagisme & 8 & 57 & 27 & 66 \\
\hline $\begin{array}{l}\text { Consommation } \\
\text { d'alcool } \\
\text { supérieure aux } \\
\text { recommandations } \\
\text { pour la } \\
\text { consommation à } \\
\text { faible risque }^{a}\end{array}$ & 2 & 14 & 8 & 20 \\
\hline \multicolumn{5}{|l|}{ Test de diagnostic } \\
\hline $\begin{array}{l}\text { Test d'antigène } \\
\text { urinaire positif }\end{array}$ & 13 & 93 & 40 & 98 \\
\hline $\begin{array}{l}\text { Culture } \\
\text { d'expectorations/ } \\
\text { LBA positive }\end{array}$ & 5 & 36 & 5 & 12 \\
\hline \multicolumn{5}{|l|}{ Gravité } \\
\hline Hospitalisation & 14 & 100 & 41 & 100 \\
\hline Admission en USI & 4 & 29 & 11 & 27 \\
\hline Intubation & 2 & 14 & 6 & 15 \\
\hline Décès & 1 & 7 & 1 & 2 \\
\hline
\end{tabular}

Abréviations : LBA, lavage bronchoalvéolaire; USI, unité de soins intensifs

a Femmes : > 10 boissons/semaine, hommes; > 15 boissons/semaine (14)
Tableau 3 : Résultats de la culture et du typage des isolats des TRE

\begin{tabular}{|c|c|c|c|c|c|c|c|c|c|}
\hline \multirow[b]{2}{*}{ TRE } & \multirow{2}{*}{$\begin{array}{l}\text { Distance } \\
\text { à de } \\
\text { l'épicentre } \\
(\mathrm{km})\end{array}$} & \multirow{2}{*}{$\begin{array}{l}\text { Résultat } \\
\text { de la } \\
\text { culture de } \\
\text { Legionella } \\
\text { (UFC/L)a }^{\text {U. }}\end{array}$} & \multicolumn{7}{|c|}{ Résultats du typage par séquence } \\
\hline & & & flaA & flaA & flaA & flaA & flaA & flaA & flaA \\
\hline$A$ & 1,2 & 10000 & 1 & 4 & 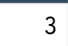 & 1 & 1 & 1 & 1 \\
\hline B & 1,5 & 10000 & ND & 14 & 16 & 16 & 15 & 13 & 2 \\
\hline C & 3,5 & 2000000 & 1 & 4 & 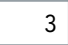 & 1 & 1 & 1 & ND \\
\hline D & 7,1 & 2000000 & 1 & 6 & 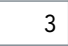 & 10 & 1 & 1 & 11 \\
\hline $\mathrm{E}$ & 7,3 & 1000000 & 11 & 14 & 16 & 12 & 15 & 13 & $z^{\prime}$ \\
\hline $\mathrm{F}$ & 7,8 & 2100000 & 1 & 4 & 3 & 1 & 1 & 1 & ND \\
\hline G & 7,9 & 3900000 & 1 & 4 & ND & ND & ND & 1 & ND \\
\hline $\mathrm{H}$ & 8,1 & 3880000 & 11 & 4 & 16 & 12 & 15 & 13 & 9 \\
\hline 1 & 8,3 & 6600000 & $\mathrm{ND}^{\mathrm{b}}$ & ND & ND & ND & ND & ND & ND \\
\hline J & 8,5 & 2000000 & $N^{c}$ & ND & ND & ND & ND & ND & ND \\
\hline K & 8,8 & 7400000 & 1 & 4 & 3 & 1 & 1 & 1 & 1 \\
\hline $\mathrm{L}$ & 9,7 & 5600000 & $N D^{d}$ & ND & ND & ND & ND & ND & ND \\
\hline M & 9,7 & 2000000 & 11 & 14 & 16 & 12 & 15 & 13 & 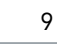 \\
\hline $\mathrm{N}$ & 10,3 & 1000000 & 1 & 4 & 3 & 1 & 1 & 1 & 1 \\
\hline O & 11,5 & 4880000 & $N^{e}$ & ND & ND & ND & ND & ND & ND \\
\hline
\end{tabular}

Abréviations : ND, non disponible; TRE, tour de refroidissement à l'eau; UFC, unités formant des colonies

Les résultats indiqués concernent l'échantillon de TRE avec une culture de Legionella quantifiable positive sur laquelle une analyse de typage a été effectuée; certaines TRE comportaient plus d'une culture de Legionella quantifiable positive

' L'isolat a été détruit par le laboratoire commercial, en violation de la réglementation provinciale qui exige que tous les isolats de Legionella provenant de TRE ayant des niveaux de 1000000 UFC/L ou plus soient conservés pendant au moins trois mois

' L'isolat n'a pas pu être analysé en raison d'une contamination

d L'isolat n'a pas été envoyé par le laboratoire commercial au laboratoire provincial de santé

publique comme l'a exigé la santé publique; il est présumé détruit ou perdu

e Lors d'une nouvelle analyse au laboratoire provincial de santé publique, l'isolat s'est révélé être du sérogroupe 2 à 15 au lieu de 1; le typage n'a donc pas été effectué

\section{Tableau 4 : Résultats du typage fondé sur le séquençage des isolats cliniques}

\begin{tabular}{|c|c|c|c|c|c|c|c|c|}
\hline \multirow{2}{*}{$\begin{array}{l}\text { Identification du } \\
\text { cas }\end{array}$} & \multirow{2}{*}{ Cadre d'exposition } & \multicolumn{7}{|c|}{ Résultats du typage fondé sur le séquençage } \\
\hline & & flaA & pilE & asd & mip & mompS & proA & neuA \\
\hline 3 & Domicile privé & ND & 9 & 2 & 5 & 3 & 20 & 15 \\
\hline 10 & Hôpital & ND & 9 & 2 & 5 & 3 & 20 & 15 \\
\hline 9 & Hôpital (même étage) & 12 & 9 & 2 & 5 & 3 & 20 & ND \\
\hline 11 & $\begin{array}{l}\text { Résidence pour personnes autonomes recevant des } \\
\text { traitements contre le cancer }\end{array}$ & 12 & ND & ND & ND & 3 & ND & ND \\
\hline 12 & Résidence privée pour aînés & 12 & 9 & 2 & 5 & 3 & 20 & 15 \\
\hline
\end{tabular}


cas : 12) était un nouveau type, ST2858, dans la base de données du Groupe de travail européen sur les infections à Legionella (EWGLI). Les résultats de typage des TRE ne correspondaient pas aux isolats cliniques (tableau 3 ).

\section{Discussion}

Nous avons enquêté sur un agrégat spatio-temporel de 14 cas de L. pneumophila de sérogroupe 1 déclarés sur une période de 12 semaines de juin à août 2019, à Montréal. L'éclosion a été initialement détectée grâce à l'analyse d'agrégations spatio-temporelles quotidienne automatisée des données locales sur les maladies à déclaration obligatoire. Notre enquête épidémiologique a révélé que les cas liés à l'éclosion avaient tendance à être plus jeunes, à présenter plus de comorbidités, notamment une immunité compromise ou supprimée, par rapport aux cas non liés à l'éclosion. Aucune exposition commune n'a été identifiée à l'exception du lieu de résidence. Deux sous-agrégats ont été identifiés : trois cas résidaient dans la même résidence privée pour aînés et quatre avaient été hospitalisés au même étage d'un hôpital pendant toute leur période d'incubation. Le typage a été effectué pour cinq cas; les résultats suggéraient une source commune pour tous les cas. L'éclosion a été déclarée terminée à la mi-octobre, mais une source environnementale n'a jamais été identifiée.

L'enquête de cette éclosion a bénéficié de l'accès à des résultats de surveillance mensuels de la $L$. pneumophila pour toutes les TRE dans le registre provincial et du prélèvement rapide d'échantillons et de la décontamination des TRE (par le biais d'ordonnances de santé publique). Si l'éclosion a finalement été maîtrisée, nos enquêtes environnementales approfondies n'ont pas permis d'identifier la source. Une explication possible est qu'une TRE peut abriter simultanément plusieurs sérogroupes de $L$. pneumophila $(15,16)$ qui peuvent évoluer rapidement dans le temps (11) (par exemple entre l'exposition du cas et le prélèvement de la TRE).

II n'est pas rare de ne pas être en mesure d'identifier la source d'une éclosion de L. pneumophila. Les Centers for Disease Control and Prevention (CDC) n'ont pas trouvé de source pour 11 des 17 éclosions de L. pneumophila de sérogroupe 1 associées à des expositions environnementales ou indéterminées à l'eau en 2013 à 2014 (17). De même, au Royaume Uni, la source a été identifiée dans moins de $50 \%$ des éclosions de Legionella $(18,19)$. Des études récentes suggèrent que le typage fondé sur le séquençage est insuffisant pour discriminer certains types de L. pneumophila du sérogroupe $1(15,20)$; il est donc possible que nos cas d'éclosion n'aient pas été infectés par la même L. pneumophila du sérogroupe 1 ; cependant, ce problème n'a pas été signalé pour $L$. pneumophila du sérogroupe 1 ST2858.
La DRSP a rencontré plusieurs défis majeurs entravant I'identification de la source de cette éclosion. Les isolats cliniques (i.e. les cultures d'expectorations) n'étaient disponibles que pour un quart des cas, ce qui a limité notre capacité à évaluer ceux qui étaient liés à l'éclosion et a brouillé la recherche d'une source commune. L'indisponibilité d'isolats environnementaux provenant de TRE avec des résultats de la surveillance de L. pneumophila inférieurs à 1000000 UFC/L, parce que les laboratoires ne sont pas légalement mandatés pour les conserver, a également entravé notre enquête. Ces TRE ont dû être rééchantillonnées, souvent quelques semaines après le résultat positif initial, parfois après l'application de mesures correctives, ce qui a réduit la probabilité d'isoler à nouveau la même $L$. pneumophila qui avait été présente pendant la période $d$ 'incubation des cas. Il existe des preuves de l'existence d'éclosions de Legionella associés à des concentrations de L. pneumophila inférieures à 1000 000 UFC/L : une revue systématique de toutes les éclosions de Legionella attribuées à des TRE en 2001 à 2012 a trouvé des concentrations de Legionella inférieures à 1000000 UFC/L dans 4 éclosions sur 19 (21). Une éclosion de légionellose de 2017 dans la région sociosanitaire de Mauricie-et-Centre-du-Québec a été génétiquement liée à une TRE avec un résultat de «flore interférente " lors de la surveillance mensuelle de la Legionella et qui avait une concentration de 630000 UFC/L de Legionella lors du rééchantillonnage (1). Étant donné que 11 des 14 patients de notre éclosion étaient immunodéprimés ou immunodéficients, il est possible que la source soit une TRE avec des niveaux de L. pneumophila de sérogroupe 1 inférieurs à 1000000 UFC/L.

Un autre défi important était le manque de bases de données complètes sur les sources potentielles d'aérosolisation de l'eau autres que les TRE, par exemple les jeux d'eau et fontaines décoratives sur les propriétés publiques et privées, les sites de construction publics et privés et les systèmes d'irrigation à grande échelle. Par conséquent, nous aurions pu négliger une source potentielle. De plus, alors que la RBQ estime que presque toutes les TRE au Québec sont enregistrées, les TRE sur les bâtiments fédéraux font l'objet d'une surveillance fédérale pour la $L$. pneumophila et les résultats ne sont pas communiqués à la RBQ.

Enfin, le protocole d'échantillonnage provincial prévoit la collecte d'un seul échantillon d'eau de $1 \mathrm{~L}$ par TRE, à la fois dans des conditions de routine et lors d'une éclosion. En revanche, les CDC recommandent d'obtenir cinq échantillons d'eau de $1 \mathrm{~L}$ et trois écouvillons de biofilm (provenant de zones précises) par TRE dans le cadre d'une enquête d'éclosion (22). Suivre le protocole d'échantillonnage des TRE des CDC aurait probablement augmenté la probabilité de détecter la L. pneumophila de sérogroupe 1 dans les TRE échantillonnées, augmentant ainsi la probabilité d'identifier la source de notre éclosion. 


\section{Conclusion}

Le présent rapport décrit une éclosion communautaire de L. pneumophila de sérogroupe 1 avec des sous-agrégats dans une résidence privée pour aînés et sur un étage d'hôpital. Alors que le typage des isolats cliniques suggérait une source environnementale commune, notre enquête n'a pas réussi à identifier cette source. De futures enquêtes $d$ ' éclosions pourraient bénéficier de la disponibilité d'un plus grand nombre $d$ 'isolats cliniques pour le typage, possiblement en ajoutant un test d'amplification des acides nucléiques (TAAN) à la culture (par exemple lorsque l'administration d'antibiotiques a déjà commencé), de registres locaux des sources d'aérosolisation d'eau autres que les TRE (e.g. les fontaines décoratives), d'un accès en continu à tous les résultats de la surveillance mensuelle des TRE, plutôt que de ne déclarer à la santé publique que les résultats dépassant un seuil, et d'un accès à tous les isolats récents de L. pneumophila obtenus par la surveillance mensuelle des TRE (quelle que soit la concentration) pour le typage. Des études additionnelles sont nécessaires pour mettre à jour et résumer les sources environnementales de L. pneumophila qui ont été liées à des cas ou des éclosions, ainsi que pour déterminer des seuils sanitaires de L. pneumophila qui tiennent compte de la possibilité que des personnes immunodéprimées ou immunodéficientes soient infectées par des concentrations plus faibles de bactéries.

\section{Déclaration des auteurs}

G. C. a codirigé l'enquête épidémiologique et est l'auteure principal du manuscrit

J. B. et F. L. ont dirigé l'enquête environnementale, ont contribué à la rédaction des sections sur l'enquête environnementale et ont fourni une rétroaction sur le manuscrit

C. L. a supervisé les analyses effectuées au laboratoire provincial de santé publique et a fourni une rétroaction sur le manuscrit P. A. P. a codirigé l'enquête épidémiologique et a fourni une rétroaction sur le manuscrit

D. K. a supervisé l'enquête environnementale et a fourni une rétroaction sur le manuscrit

E. L. a supervisé l'enquête épidémiologique et a fourni une rétroaction sur le manuscrit

\section{Intérêts concurrents}

Aucun.

\section{Remerciements}

Les auteurs tiennent à remercier tous les membres des équipes de Prévention et contrôle des maladies infectieuses (PCMI) et Environnement urbain et saines habitudes de vie (EUSHV) de la Direction régionale de santé publique de Montréal, ainsi que des Activités courantes et vigie sanitaire (ACVS) qui ont contribué à la gestion de cette éclosion. Les auteurs tiennent également à remercier le Laboratoire de santé publique du Québec (LSPQ), le Centre d'expertise en analyses environnementales du Québec (CEAEQ), la Régie du bâtiment du Québec (RBQ), l'équipe de prévention et de contrôle des infections de l'hôpital et les administrateurs des résidences privées pour aînés qui ont été impliqués dans la gestion de cette éclosion.

\section{Financement}

Tous les auteurs ont reçu une aide salariale de leurs organisations respectives pour réaliser ce travail.

\section{Références}

1. Leblanc M, Markowski F, Menard S, Boivin S, Marcoux-Huard C, Chartrand J. Vigie - Interventions: la légionellose. Flash Vigie 2018;13(4):1-7.

2. Lévesque $\mathrm{S}$, Plante $\mathrm{PL}$, Mendis N, Cantin P, Marchand G, Charest H, Raymond F, Huot C, Goupil-Sormany I, Desbiens F, Faucher SP, Corbeil J, Tremblay C. Genomic characterization of a large outbreak of Legionella pneumophila serogroup 1 strains in Quebec City, 2012. PLoS One 2014 Aug;9(8):e103852. DOI PubMed

3. Ministère de la santé et des services sociaux. Guide d'intervention - La légionellose (Édition 2015). Quebec City (QC): Ministère de la santé et des services sociaux; 2015 (accédé 2019-10-12). https://publications.msss.gouv.qc.ca/ msss/document-000776/

4. Robitaille M. Protocole d'échantillonnage de l'eau du circuit des tours de refroidissement pour la recherche des légionelles (DR-09-11). Quebec City (OC): Centre d'expertise en analyse environnementale du Québec; 2013 (accédé 2020-09-19). http://www.ceaeq.gouv.qc.ca/ documents/publications/echantillonnage/DR09_11echant_ tours.pdf

5. Régie du bâtiment du Québec. Règlement modifiant le Code de sécurité intégrant des dispositions relatives à l'entretien d'une installation de tour de refroidissement à l'eau. Gazette officielle du Québec 22 (accédé 201912-10). http://www2.publicationsduquebec.gouv.qc.ca/ dynamicSearch/telecharge.php?type=1\&file=61543.pdf

6. Racine $\mathrm{P}$, Elliott $\mathrm{S}$, Betts $\mathrm{S}$. Legionella regulation, cooling tower positivity and water quality in the Quebec context (AT-19-C042). ASHRAE Trans 2019;125(Part 1):350-9.

7. Prussin AJ 2nd, Schwake DO, Marr LC. Ten questions concerning the aerosolization and transmission of Legionella in the built environment. Build Environ 2017 Oct;123: 684-95. DOI PubMed

8. Knox NC, Weedmark KA, Conly J, Ensminger AW, Hosein FS, Drews SJ; Legionella Outbreak Investigative Team. Unusual Legionnaires' outbreak in cool, dry Western Canada: an investigation using genomic epidemiology. Epidemiol Infect 2017 Jan;145(2):254-65. DOI PubMed 
9. UK Health and Safety Executive. HSG274: Legionnaires' disease: technical guidance: Part 3: The control of legionella bacteria in other risk systems. 2013 (accédé 2019-10-12). http://www.hse.gov.uk/pubns/priced/hsg274part3.pdf

10. van Heijnsbergen E, Schalk JA, Euser SM, Brandsema PS, den Boer JW, de Roda Husman AM. Confirmed and potential sources of Legionella reviewed. Environ Sci Technol 2015;49(8):4797-815. DOI PubMed

11. National Academies of Sciences. Engineering and Medicine. Management of legionella in water systems. Washington (DC): The National Academies Press; 2019.

12. Gaia V, Fry NK, Afshar B, Lück PC, Meugnier H, Etienne J, Peduzzi R, Harrison TG. Consensus sequence-based scheme for epidemiological typing of clinical and environmental isolates of Legionella pneumophila. J Clin Microbiol 2005;43(5):2047-52. DOI PubMed

13. Ratzow S, Gaia V, Helbig JH, Fry NK, Lück PC. Addition of neuA, the gene encoding $\mathrm{N}$-acylneuraminate cytidylyl transferase, increases the discriminatory ability of the consensus sequence-based scheme for typing Legionella pneumophila serogroup 1 strains. J Clin Microbiol 2007 Jun;45(6):1965-8. DOl PubMed

14. Butt P, Beirness D, Gliksman L, Paradis C, Stockwell T. L'alcool et la santé au Canada : Résumé des données probantes et directives de consommation à faible risque. Ottawa $(\mathrm{ON})$ : Centre canadien sur les dépendances et l'usage de substances; 2011 (accédé 2020-04-13). https://www.ccsa.ca/fr/lalcool-et-la-sante-au-canada-resumedes-donnees-probantes-et-directives-de-consommationfaible

15. Wüthrich D, Gautsch S, Spieler-Denz R, Dubuis O, Gaia V, Moran-Gilad J, Hinic V, Seth-Smith HM, Nickel CH, Tschudin-Sutter S, Bassetti S, Haenggi M, Brodmann P, Fuchs S, Egli A. Air-conditioner cooling towers as complex reservoirs and continuous source of Legionella pneumophila infection evidenced by a genomic analysis study in 2017, Switzerland. Euro Surveill 2019 Jan;24(4):1800192. DOI PubMed

16. Lapierre P, Nazarian E, Zhu Y, Wroblewski D, Saylors A, Passaretti T, Hughes S, Tran A, Lin Y, Kornblum J, Morrison SS, Mercante JW, Fitzhenry R, Weiss D, Raphael BH, Varma JK, Zucker HA, Rakeman JL, Musser KA. Legionnaires' disease outbreak caused by endemic strain of Legionella pneumophila, New York, New York, USA, 2015. Emerg Infect Dis 2017 Nov;23(11):1784-91. DOI PubMed
17. McClung RP, Roth DM, Vigar M, Roberts VA, Kahler AM, Cooley LA, Hilborn ED, Wade TJ, Fullerton KE, Yoder JS, Hill VR. Waterborne disease outbreaks associated with environmental and undetermined exposures to water United States, 2013-2014. MMWR Morb Mortal Wkly Rep 2017 Nov;66(44):1222-5. DOI PubMed

18. Lock K, Millett C, Heathcock R, Joseph CA, Harrison TG, Lee JV, Rao G, Surman-Lee S; Outbreak Control Team. Public health and economic costs of investigating a suspected outbreak of Legionnaires' disease. Epidemiol Infect 2008 Oct;136(10):1306-14. DOI PubMed

19. Lee S, Lee J. Outbreak investigations and identification of legionella in contaminated water. Methods Mol Biol 2013;954:87-118. DOI PubMed

20. Buultjens $A H$, Chua KY, Baines SL, Kwong J, Gao W, Cutcher Z, Adcock S, Ballard S, Schultz MB, Tomita T, Subasinghe N, Carter GP, Pidot SJ, Franklin L, Seemann T, Gonçalves Da Silva A, Howden BP, Stinear TP. A supervised statistical learning approach for accurate Legionella pneumophila source attribution during outbreaks. Appl Environ Microbiol 2017 Oct;83(21):e01482-17. DOl PubMed

21. Walser SM, Gerstner DG, Brenner B, Höller C, Liebl B, Herr CE. Assessing the environmental health relevance of cooling towers--a systematic review of legionellosis outbreaks. Int J Hyg Environ Health 2014;217(2-3):145-54. DOI PubMed

22. Centers for Disease Control and Prevention. Sampling procedure and potential sampling sites: protocol for collecting environmental samples for Legionella culture during a cluster or outbreak investigation or when cases of disease may be associated with a facility. Atlanta (GA): CDC; 2019 (accédé 2019-12-04). https://www.cdc.gov/legionella/ downloads/cdc-sampling-procedure.pdf 